\title{
An Efficient Approach for Content based Image Retrieval using SVM, KNN-GA as Multilayer Classifier
}

\author{
Vinay Kumar Lowanshi \\ Department of Computer \\ Science and Engineering \\ LNCT, Bhopal (India)
}

\author{
Shweta Shrivastava \\ Department of Computer \\ Science and Engineering \\ LNCT, Bhopal (India)
}

\author{
Vineet Richhariya, Ph.D \\ Department of Computer \\ Science and Engineering \\ LNCT ,Bhopal (India)
}

\begin{abstract}
The technology is growing day by day in various fields and image retrieval is one of the most of them, it is more interesting and fastest growing research areas. It is an effective and efficient tool for managing large image databases. In most Content-Based Image Retrieval (CBIR) systems, images are represented and differentiated by a set of low-level visual features; hence a direct correlation with highlevel semantic information will be absent. Therefore, a gap exists between high-level information. In this paper they proposed novel approach for content based image retrieval was two tier architecture model is used for most accurate retrieval. In the first tier first feature extraction process done using PSO with SVM classifier, after successful classification in first tier the retrieved result has been passed into the second tier classifier. And in the second tier KNN classifier is used but as they knew that GA is one of the optimization technique and it produces the best optimized result in maximum cases so it is applied with the KNN classifier, and it produces more accurate and efficient compared result.
\end{abstract}

\section{Keywords}

Content based image retrieval (CBIR), feature extraction, SVM, PSO, KNN, GA, object optimization.

\section{INTRODUCTION}

In the content based image retrieval the semantic gap between pre-processed stored images and query image reduces. In image retrieval, basically image is retrieved based on three attributes such as color, texture and shape. CBIR is a technique in which search for images from large image database takes place based on image content itself when a request is given by the user. The two main steps in a CBIR technique are feature extraction and similarity measurement. Various algorithms used for describing color, shape and texture.

CBIR follows two major steps while processing, they are feature extraction and similarity measurement between two extracted features. Commonly used content based features or the low level features are color, texture and shape [1].

In general features can be classified into color, texture and shape and in domain specific - face, fingerprint etc features as they depend on application. As image perception differs for different person, there is no single best feature representation. A given feature has got multiple representations that characterize the feature from different perspective. Instead of exact matching, content based image retrieval calculates visual similarities as well as feature based similarities between a query image and database images. Accordingly, the retrieval result is not a single image but a list of images ranked by their similarities with the query image. Many similarity measures have been developed for image retrieval based on empirical estimates of the distribution of features in recent years. Different similarity and distance measures will affect retrieval performances of an image retrieval system significantly. Various similarity measurement mathematic formulas are Minkowski-Form distance; Quadratic Form distance, Mahalanobis distance etc. can be used to measurement of distance between features.

The CBIR has many applications areas such as architectural design, mining, education, commerce, military, medical diagnosis, biomedical and web image classification. All most of The CBIR research is finding technique to measure the performance for retrieving more similar image from the image databases of retrieval.

In this paper section 2 describe the literature of various techniques suggested by different researchers, section 3 defines the image retrieval with different techniques and section $4 \& 5$ described the proposed methodology with black diagram and experimental result analysis respectively, then section 6 concludes the summery of the work with its future scope.

\section{LITERATURE REVIEW}

In [3] paper, they proposed the design and improvement of multi-tiered content-based image retrieval (CBIR) structure for infinitesimal images utilizing a reference database that restrain images of other than one disease. The proposed CBIR structure uses a multi-tiered scheme to categorize and repossess microscopic images involving their explicit subtypes, which are mostly complicated to discriminate and categorize. This structure facilitates both multi-image query and slide-level image retrieval in order to safeguard the semantic consistency among the retrieved images. Novel weighting terms stimulated from information retrieval supposition are described for multiple-image query and retrieval. The performance of the design was tested on a dataset including 1666 imaged high power fields mined from 57 follicular lymphoma tissue slides with three sub-types and 44 neuro-blastoma tissue slides with four sub-types. All slides are semantically annotated according to their sub-types by skilled pathologists. Upon using leave one slide out testing method the multi-image query algorithm with the proposed weighting design achieves estimate $93 \%$ and $86 \%$ of standard categorization precision at the first level retrieval outperforming the image-level retrieval precision by about 38 and 26 percentage points for follicular lymphoma (FL) and neuro-blastoma (NB) diseases correspondingly.

In [4] paper, they proposed high level filtering to avoid where we are using the Anisotropic Morphological Filters, hierarchical Kaman filter and particle filter proceeding with feature extraction method based on color and gray level feature and after this the results were normalized. 
In [5] paper, they are concentrating on CBIR and fundamental concepts pertaining to it as well as Relevance Feedback and its assorted mechanisms. Relevance Feedback (RF) was integrated to CBIR systems. By permitting the user to assess iteratively the answers as appropriate/inappropriate or even giving him/her the chances to indicate a degree of relevance (user's feedbacks), the structure creates a new query that better detains the user's needs and hence raising the chances to get more pertinent image results. An important contribution in this work is a relative analysis of CBIR systems using reference feedback.

In [6] paper, they proposed an improved PSO method with adaptive weight of SVM to get better retrieval performance. Together with, a composite histogram method is working which corresponds to a composition of color, luminance, and edge features in the image, to extract low-level feature of an image. As a result, every image can be presented as a composite histogram. The experimental results show that the PSO-SVM method for image retrieval and clustering problem can achieve higher recognition accuracy and higher recognition speed.

In [7] paper, they attempts to study and provides a brief knowledge about the different image classification approaches and different classification methods. Most common approaches for image classification can be categories as supervised and unsupervised, or parametric and nonparametric or object-oriented, sub pixel, per-pixel and per field or spectral classifiers, contextual classifiers and spectralcontextual classifiers or hard and soft classification. This survey gives theoretical knowledge about different classification methods and provides the advantages and disadvantages of various classification methods.

In [8] paper, they presented a fresh proposal for image retrieval based on two-level architecture stimulated from human cognitive mechanisms. These two levels make available both generic resemblance and semantic information related to unique characteristics. Even though the proposed architecture can be customized for any content-based image retrieval relevance, the work is focused on medical images and more exclusively on brain MRI data. The key impetus is that in medical applications, it is vital to be proficient to coalesce in an efficient way, image resemblance with precise, semantics related to pathology in order to make available the user with relevant cases and support diagnosis. A depiction of the architecture and function of the proposed CBIR proposal is presented as well as explicit details for the relevance to brain MRI retrieval.

In [14] paper, they proposed an interactive design for regionbased image clustering and retrieval. By performing clustering prior to image retrieval, the search space can be reduced to those clusters that are nearest to the query target. Primary, the image is segmented to regions by using an unsupervised segmentation method. This is an area where a enormous number of regions are involved. To reduce search space for region-based image retrieval, we use clustering based on genetic algorithm. Fuzzy resemblance is used in order to compute the similarity of two images. Moreover, a two-class SVM is trained based on user interests to advance image retrieval. Experiments were performed on COREL image database and show the effectiveness of the proposed approach.

In [15] proposed the usefulness of pertaining genetic algorithm (GA)-based attribute subset assortment and constraint optimization of support vector machine (SVM) for content-based image retrieval (CBIR). The SVM is one of the innovative techniques for outline categorization and it has been comprehensively used in many relevance areas. The kernel factors setting for SVM in the training progression impacts on the categorization precision. Attribute subset selection is an additional factor that impacts categorization precision. The objective is to concurrently optimize the parameters and attribute subset without degrading the SVM categorization precision using the GA-based method for CBIR. In this, we show that the proposed GA-based method outperforms SVM to the difficulty of the image categorization problem in CBIR. It is compared with $\mathrm{NN}$ and SVM algorithm and the proposed GA-based method extensively improves the categorization precision and has fewer input attribute for SVM.

\section{IMAGE RETRIEVAL TECHNIQUES}

In this section we briefly discuss about the all four methods of image classification i.e. RF-SVM, NB-NN, Fuzzy and BMMA. First subsection defines about the RF-SVM and its advantages and disadvantages. Second subsection defines about the NB-NN and its advantages and disadvantages. Third subsection defines about the Fuzzy and its advantages and disadvantages. Forth subsection defines about the BMMA and its advantages and disadvantages.

\subsection{Image retrieval using Relevance Feedback}

A major task in the CBIR systems is the similarity matching between the query image and the retrieved images. Unfortunately, the gap between high-level concepts and lowlevel features, as well as the subjective perception for the visual content by the human beings, result a significant mismatch between the retrieval results judged manually and by the computers. To improve the retrieval precision, human interactions are usually involved. Relevance feedback is an interactive process which integrates ,users ${ }^{e e}$ evaluation of the retrieval results. Typically, the relevance feedback technique includes an interactive scoring system to evaluate the past retrieval results to improve the subsequent content retrieval [10].

\subsection{Image Retrieval using NF}

The technique of the proposed neuro fuzzy (NF) content based image retrieval system in two stages. Stage 1: the query to regain the images from database is organized in terms of natural language such as typically content, more content and few content of some explicit color. Fuzzy logic is used to identify the query [9].

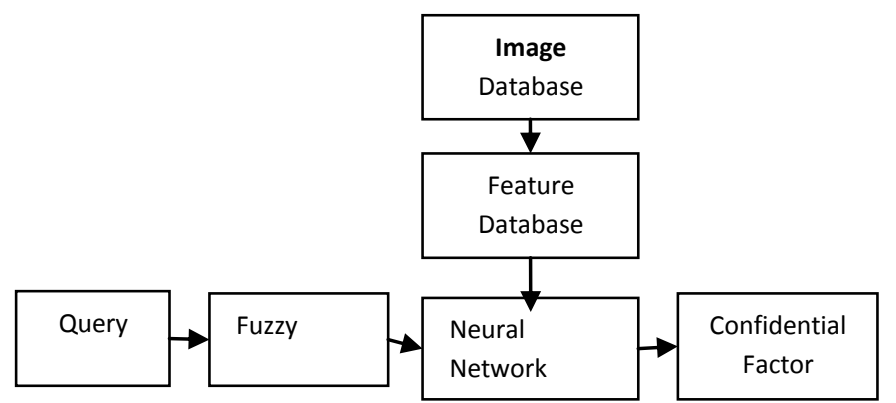

Fig. 1: Block diagram of Neuro Fuzzy system [9]

\subsection{Image retrieval using DWT}

DWT [11] divides an image into 4 multi-resolution sub-bands. At level 1, DWT decomposes image into four nonoverlapping sub-bands: LL1 (Approximate sub band), HL1 
(Horizontal sub band), LH1 (Vertical sub band) and HH1 (Diagonal Sub band). Here, LL1 is low frequency component whereas HL1, LH1 and HH1 are high frequency (detail) components. Embedding watermark in low frequency coefficients can increase robustness significantly but maximum energy of most of the natural images is concentrated in approximate (LL) sub-band. Hence modification in this low frequency sub band will cause severe and unacceptable image degradation. Hence watermark is not embedded in LL sub band. The good areas for watermark embedding are high frequency sub bands (HL, $\mathrm{LH}$ and $\mathrm{HH}$ ), because human naked eyes are not sensitive to these sub bands. Thus we have selected HH1 band to embed watermark.

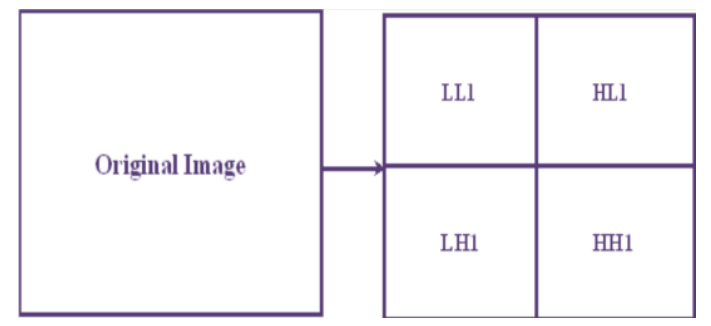

Figure 2: 1 Level DWT

\subsection{Image retrieval using Genetic \\ algorithm}

Genetic programming (GP) [2], such as other evolutionary computation algorithms, is an artificial intelligence problemsolving technique based on the principles of biological inheritance and evolution. In GP approach, the individuals represent programs that undergo evolution. The fitness evaluation consists in executing these programs, and measuring their degrees of evolution. Genetic programming, then, involves an evolution-directed search in the space of possible computer programs that best solve a given problem. At beginning of the evolution, an initial population of individuals is created. Next, loops of successive steps are performed to evolve these individuals: the fitness calculation of each individual, the selection of the individuals, based on their fitness, to breed a new population by applying genetic operators. In the following, these steps are presented in more details. Usually, a GP individual represents a program and is encoded in a tree. In this encoding, an individual contains two kinds of nodes, terminals (leaf nodes) and functions (intern nodes). Terminals are usually program inputs, although they may also be constants. Functions take inputs and produce outputs. A function input can be either a terminal or the output of another function. The fitness of an individual is determined by its effectiveness in producing the correct outputs for all cases in a training set. The training set is a set containing inputs and their correspondent previously known outputs.

To evolve the population, and optimize the desired objectives, it is necessary to choose the correct individuals to be subject to genetic operators. Thus, selection operators are employed to select the individuals, usually, based on their fitness. Examples of selection method are roulette wheel, tournament and rank-based selections. Genetic operators introduce variability in the individuals and make evolution possible, which may produce better individuals in posterior generations. The crossover operator exchanges sub-trees from a pair of individuals, generating two others. Mutation operator replaces a randomly chosen sub-tree from an individual by a sub-tree randomly generated. The reproduction operator simply copies individuals and insert them in the next generation.

\section{PROPOSED METHODOLOGY}

In proposed scheme we follows the two level classification architecture for more improvement purpose of the result, where SVM classifier is used in first level classification and KNN with GA used as second classifier which takes input from the first processed classifier.

In the algorithm follows steps are:

1. Verify image database

2. Read all training images from image database

3. Extract images RGB feature

4. Stored all extracted features in a separate single files each

5. Now read query image

6. Go to step 3, 4

7. Now apply Tier 1: SVMPSO approach for training then classification of images in separate group, apply linear Kernel function

8. Then again extract feature on bases of $\mathrm{CCM} / \mathrm{CCH}$

9. Now apply modified KNN classifier for classification of images, for best optimized retrieval result.

10. Calculate Distance: Mahalanobis distances between the feature vector of query image and the feature vectors of database images.

$$
d_{i j}=\left(\left(\overline{\mathrm{X}}_{\mathrm{i}}-\overline{\mathrm{X}}_{\mathrm{j}}\right)^{\mathrm{T}} \mathrm{S}^{-1}\left(\overline{\mathrm{X}}_{\mathrm{i}}-\overline{\mathrm{X}}_{\mathrm{j}}\right)\right)^{1 / 2}
$$

\section{Feature Extraction}

Input images will be divided into $\mathrm{M} * \mathrm{~S}$ grids before. All grids are input to extract the color feature. First, the module compute the average RGB value of the $\mathrm{M} * \mathrm{M}$ grids. Second, the inside $S^{*} S$ grids in every $M^{*} S$ grids will also be input to calculate the average RGB value. The $S * S$ grids' detail RGB information is append after the $\mathrm{M} * \mathrm{~S}$ grids' color feature information. All those are prepared for first tier SVM clustering.

\section{$\mathrm{CCH}$ feature}

$\mathrm{CCH}$ is to use find out the important feature points. All points are detected for preparing the input data of the next nearest feature and SVM classifying. The information of $\mathrm{CCH}$ feature points, including the $\mathrm{m} \times \mathrm{n}$ dim data, combines with the nearest result and it will become input for the 2-tier KNNGA classifier.

\section{Tier 1 Algorithm:}

Initialize:

Max-iterations, Particles, Dim

for $i=1$ :Particles

for $\mathrm{j}=1$ :Dim

Pos_partical $(i, j)=$ rand $* 10$;

velocity_partical $(\mathrm{i}, \mathrm{j})=$ rand $* 400$; end

$$
\text { p_best }(i, j)=\text { Pos_partical(i,j); }
$$

end

for count $=1$ :Particles

p_best_fitness $($ count $)=-400$;

end

for count $=1$ :max_iterations

for count_x $=1$ :Particles

$\mathrm{x}=$ Pos_partical(count_x,1);

$\mathrm{y}=$ Pos_partical $($ count_x,2);

ker = '@linearKernel';

global $\mathrm{p} 1 ; \mathrm{p} 1=\mathrm{x} ; \mathrm{S}=\mathrm{y} ; \operatorname{trn} \mathrm{X}=\mathrm{X} ; \operatorname{trn} \mathrm{Y}=\mathrm{Y} ; \operatorname{tst} \mathrm{X}=\mathrm{X}^{\prime}$ tst $Y=Y^{\prime}$ 
$[\mathrm{nsv}$, alpha,bias $]=\operatorname{svmTrain}(\operatorname{trnX}, \operatorname{trnY}, \mathrm{S}) ;$

actfunc $=0$;

predicted $Y=$

svcoutput(trnX,trnY,tstX,ker,alpha,bias,actfunc);

$$
\begin{aligned}
& \text { Result }=\sim \operatorname{abs}(\text { predictedY }) \\
& \text { Percent }=\operatorname{sum}(\text { Result }) / \text { length }(\text { Result }) \\
& \text { soln }=1 \text {-Percent } \\
& \quad \text { if soln } \sim=0
\end{aligned}
$$$$
\text { current_fitness }(\text { count_x })=1 / \text { abs }(\text { soln })+0.0001 \text {; }
$$$$
\text { else }
$$$$
\text { current_fitness(count_x) }=400 \text {; }
$$$$
\text { end }
$$

Proceed Result, and best fitness for next classifier end

\section{Tier 2 Algorithm: KNN}

- Supervised classification method

- Training process on selected pre-classified result

- Stores all the best training points.

- Classification starts for other new improved result with GA optimization.

- Look up its $\mathrm{k}$ nearest points and then label the new point according to which set contains the majority of its $\mathrm{k}$ neighbors and optimized with GA.

- Count the final retrieved images result.

- Apply sort function to arrange image according to its distance.

- Display the final retrieved result.

In the figure 3, its shows that the flow diagram of the proposed methodology, where it starts from the image database and query image process in parallel after that feature extraction process starts, and other further level are clearly describing the flow of the proposed methodology.

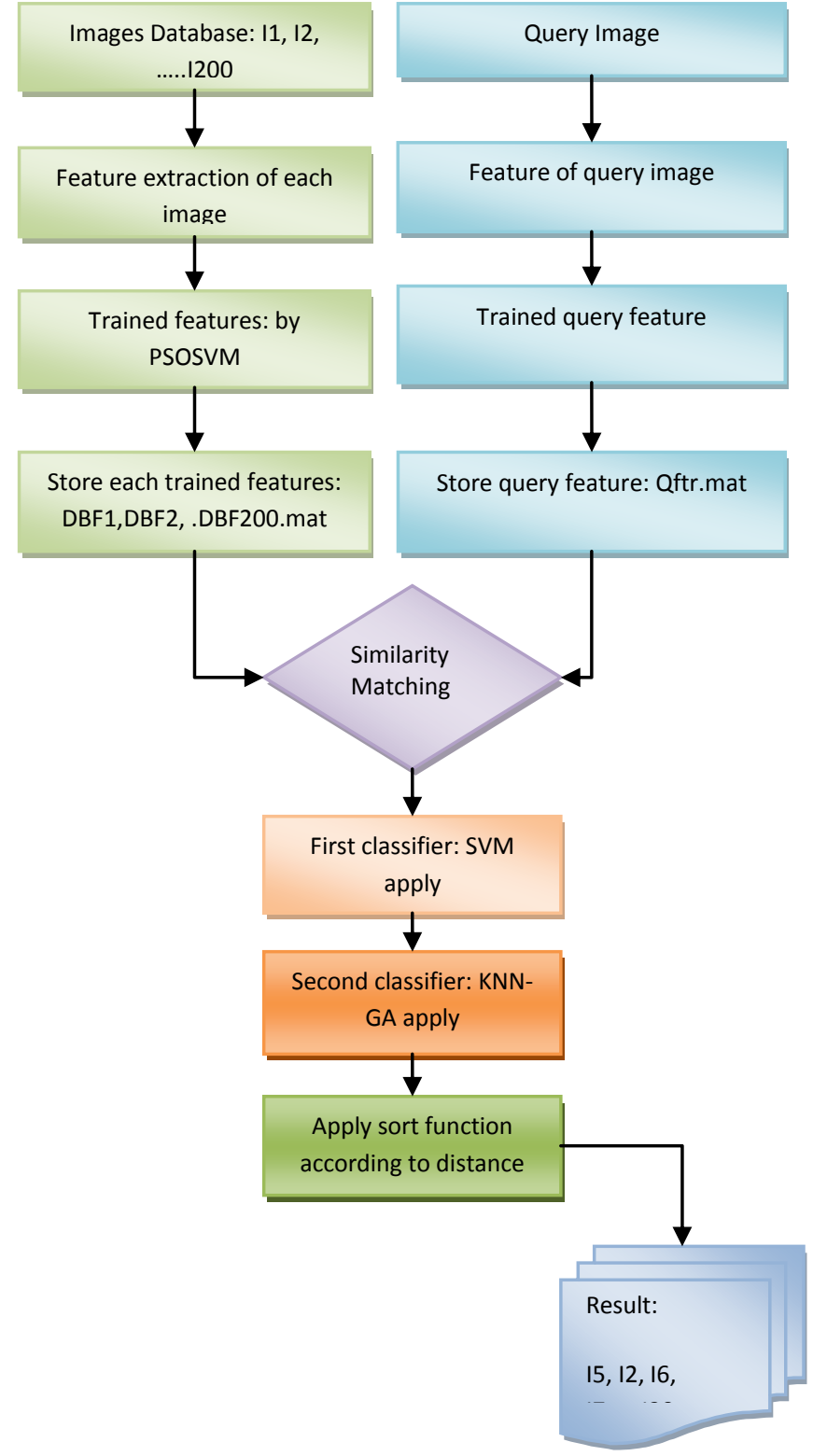

Figure 3: Block diagram of proposed methodology

\section{EXPERIMENTAL RESULTS}

To investigate the effectiveness of the proposed method of content based image retrieval. We used MATLAB software 7.8.0 and some reputed image dataset used for experimental task.

\section{Image Data Set}

The coral image data set is very famous image data set for research purpose for image retrieval. In this data set 1000 image are available, but they used only 200-300 images for different level of investigation process.

This image data set is shown in figure 4, 5, 6 compared with multi query image retrieval method and our proposed method 2-tier image retrieval. 


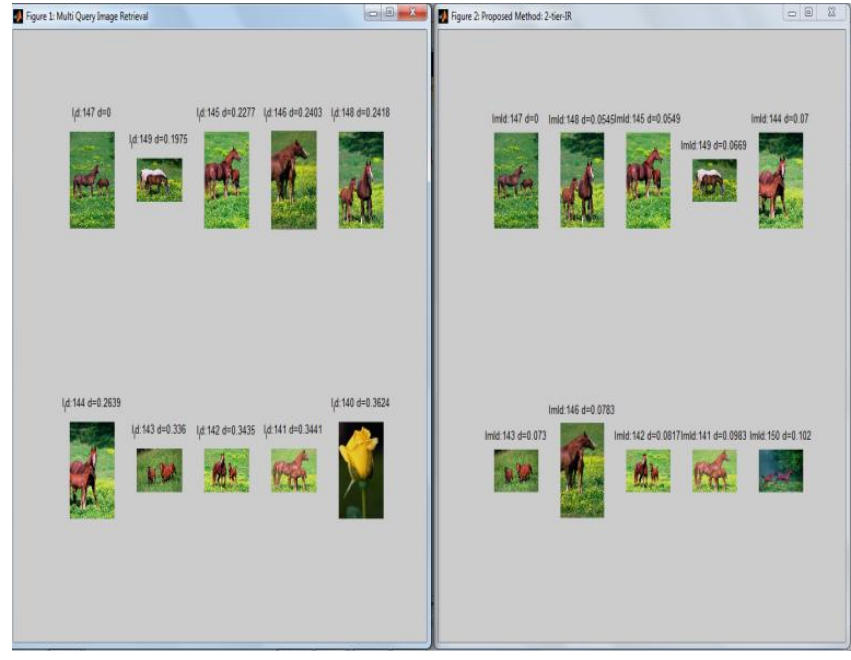

Figure 4: Compared result for horse Image

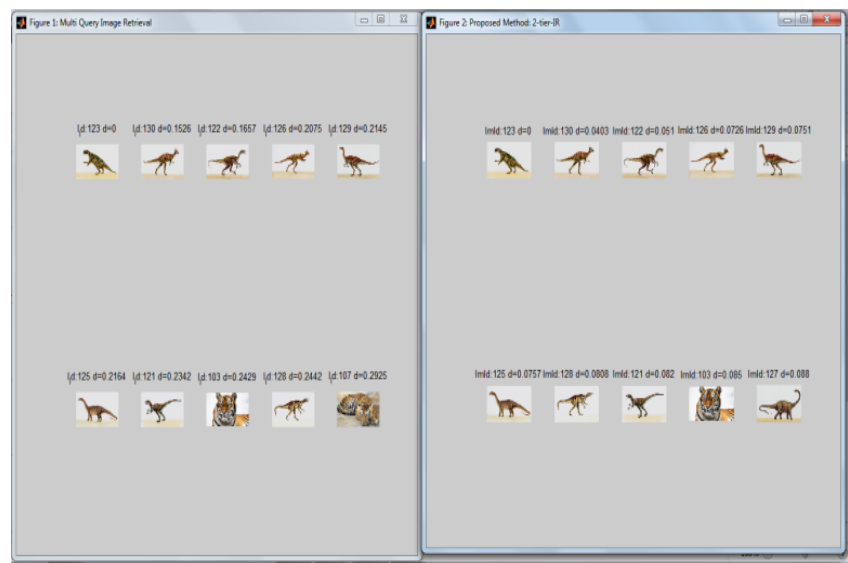

Figure 5: Compared result for dinosaur Image

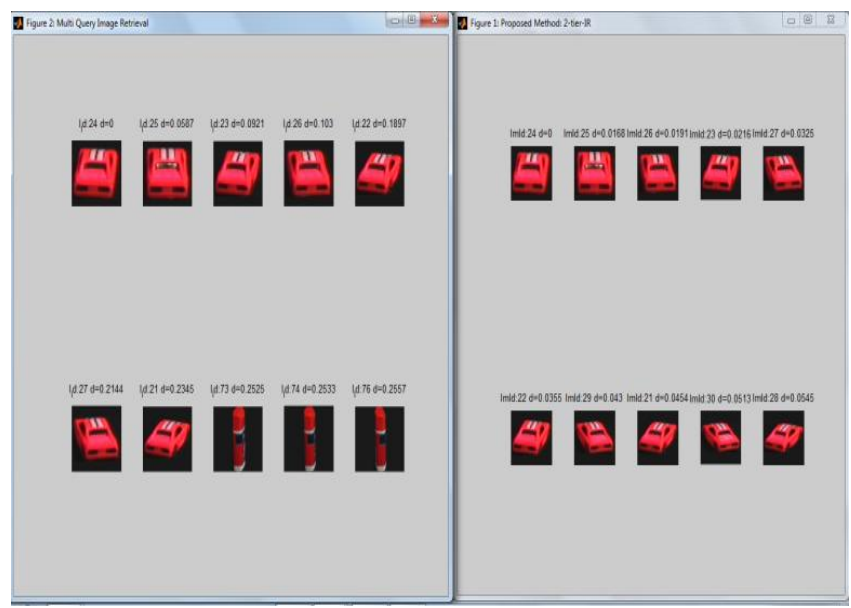

Figure 6: Compared result for car image

The image dataset are classified into different image classes from class 1 to class 15 which shows the individual class wise accuracy of mages. In this result table1 shows the accuracy of proposed method 2tier-IR is much better than MQ-IR (multiquery image retrieval) and class wise accuracy shown in table 1 and its graph is shown in figure 7. The overall accuracy is shown in table 2 and its graphical representation is shown in figure 8. The comparison between MQ-IR and 2tier-IR in which the proposed method (2tier-IR) gives the $97.33 \%$ while MQ-IR gives overall accuracy $83.33 \%$.
Table 1: Class wise accuracy of different image classes between MQ-IR \& 2tier-IR

\begin{tabular}{|l|l|l|}
\hline \multicolumn{3}{|c|}{ Class wise Accuracy } \\
\hline Image Class & MQ-IR & 2tier-IR \\
\hline Class1 & 100 & 100 \\
\hline Class2 & 100 & 100 \\
\hline Class3 & 70 & 100 \\
\hline Class4 & 100 & 100 \\
\hline Class5 & 100 & 100 \\
\hline Class6 & 100 & 100 \\
\hline Class7 & 60 & 100 \\
\hline Class8 & 90 & 100 \\
\hline Class9 & 100 & 100 \\
\hline Class10 & 80 & 100 \\
\hline Class11 & 70 & 90 \\
\hline Class12 & 20 & 80 \\
\hline Class13 & 80 & 90 \\
\hline Class14 & 90 & 100 \\
\hline Class15 & 90 & 100 \\
\hline
\end{tabular}

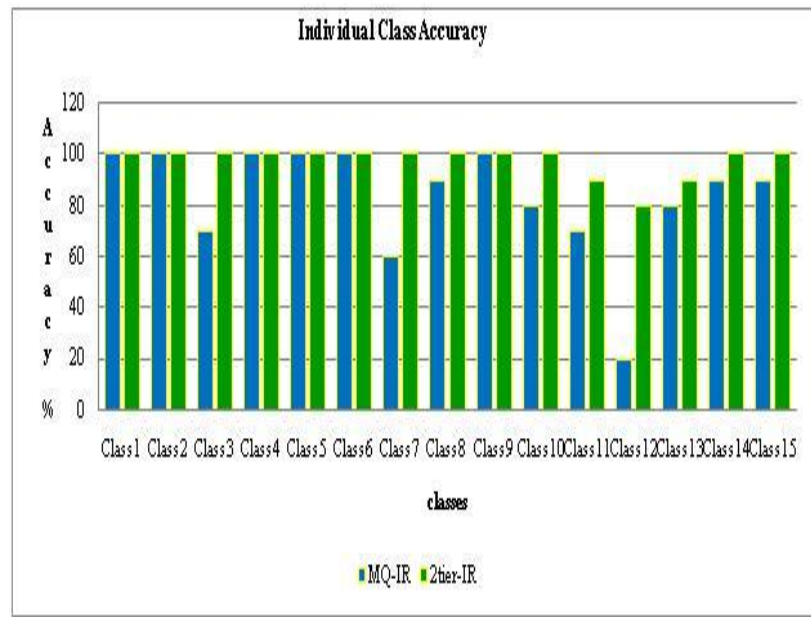

Figure 7: class wise accuracy

Table 2: Overall accuracy of Image classes

\begin{tabular}{|c|c|}
\hline \multicolumn{2}{|c|}{ Overall Accuracy } \\
\hline MQ-IR & 2 tier-IR \\
\hline 83.33 & 97.33 \\
\hline
\end{tabular}

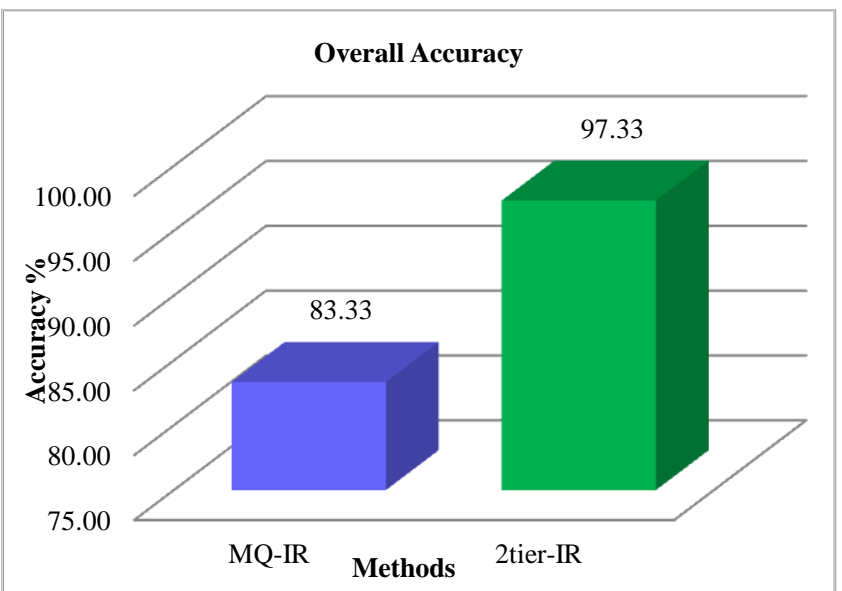

Figure 8: overall accuracy result of MQ-IR \& 2 tier-IR 


\section{CONCLUSION}

In this paper they proposed image retrieval method using modified SVM and hybrid genetic algorithm (GA) to enhance the retrieval rate of similar images, there are layered wise architecture for image retrieval, in the first layer hybrid SVM use for the classification with PSO optimizer after that the retrieved result process to the layer second which consist $\mathrm{KNN}$ with genetic algorithm, in the second layer KNN classify the more accurate result and GA enhance the better optimize retrieval result. here they have developed one existing method and it is compared with the proposed methodology and they observe that the quality parameter compared class wise accuracy and overall accuracy, and it state that the accuracy of proposed approach is $97.33 \%$ and existing is $83.33 \%$ on the same dataset.

In future the proposed methodology can be modified using some other best optimizer and to be use for the online retrieval of similar kind of data and market survey; the approach is also can be beneficial for product based searching in e-commerce market.

\section{REFERENCE}

[1] Ying Liua, Dengsheng Zhang, Guojun Lu,Wei-Ying Ma, "A survey of content-based image retrieval with highlevel semantics" Pattern Recognition 40 (2007) 262282.

[2] Jefersson Alex dos Santos, Cristiano Dalmaschio Ferreira, and Ricardo da Silva Torres, "A Genetic Programming Approach for Relevance Feedback in Region-based Image Retrieval Systems",

[3] Hatice Cinar Akakin and Metin N. Gurcan, "ContentBased Microscopic Image Retrieval System for MultiImage Queries", IEEE transactions on information technology in biomedicine, vol. 16, no. 4, July 2012

[4] S. Manoharan, S. Sathappan, "a novel approach for content based Image retrieval using hybrid filter Techniques", the 8th international conference on Computer science \& education (ICCSE 2013) April 2628, 2013. Colombo, Sri lanka.

[5] Khadidja Belattar, Sihem Mostefai "CBIR using Relevance Feedback:Comparative Analysis and Major Challenges" Computer Science Department MISC Laboratory Mentouri University Constantine , Algeria, 5th International Conference on Computer Science and Information Technology 2013.
[6] Lianze Ma, Lin Lin, Mitsuo G, "A PSO-SVM Approach for Image Retrieval and Clustering", Proceedings of the 41st International Conference on Computers \& Industrial Engineering.

[7] Pooja Kamavisdar, Sonam Saluja, Sonu Agrawal. "A survey on image classification approaches and techniques", Department of Computer Science \& Applications, SSCST, Bhilai, India, IJARCCE, Vol.2, Issue.1, Jan 2013.

[8] John Moustakas, Kostas Marias, Socrates Dimitriadis, Stelios C. Orphanoudakis, "A Two-Level Cbir Platform with Application to Brain MRI Retrieval"

[9] S. Kulkarni, B. Verma1, P. Sharma and H. Selvaraj "Content Based Image Retrieval using a Neuro-Fuzzy Technique" 2005.

[10] Mohd. Danish, Ritika Rawat, Ratika Sharma, "A Survey: Content Based Image Retrieval Based On Color, Texture, Shape \& Neuro Fuzzy", Int. Journal of Engineering Research and Applications ISSN: 2248-9622, Vol. 3, Issue 5, Sep-Oct 2013, Pp.839-844

[11] Jaya Jeswani1, Tanuja Sarode2, "A Hybrid DCT and DWT Color Image Watermarking in RGB Color Space", International Journal of Computer Science and Information Technologies, Vol. 5 (3), 2014, 3132 - 3138

[12] T. B"ack, D. B. Fogel, and Z. Michalewicz, "Evolutionary Computation 1 Basics Algorithm and Operators" Institute of Physics Publishing, 2002.

[13] Saurabh Agrawal, Nishchal K Verma, Prateek Tamrakar, Pradip Sircar, "Content based color image classification using SVM", Department of Electrical Engg., IIT, Kanpur, India, 2011 8th international conference on information technology.

[14] Fakouri R, Zamani, B. ; Fathy, M. ; Minaei, B., "RegionBased Image Clustering and Retrieval Using Fuzzy Similarity and Relevance Feedback", International Conference on Computer and Electrical Engineering, 2008, Page(s):383 - 387Print ISBN:978-0-7695-3504-3

[15] Kwang-Kyu Seo, "A GA-Based Feature Subset Selection and Parameter Optimization of Support Vector Machine for Content - Based Image Retrieval", Advanced Data Mining and Applications Lecture Notes in Computer Science Volume 4632, 2007, pp 594-604. 